\title{
The influence of Fusen herbal tea for Rhubarb in spleen deficiency rats induced by gastrointestinal tissue
}

\author{
Ting Wang ${ }^{1, a}$, Mingsan Miao ${ }^{1,{ }^{*}}$, Bai Ming ${ }^{1}$, Zuhong Wang ${ }^{2}$, Li Huan ${ }^{1}$ \\ ${ }^{1}$ Henan University of Traditional Chinese Medicine, Zhengzhou,450000,China \\ ${ }^{2}$ Fusen pharmaceutical, Nanyang, 473000, China \\ aemail: wangtingyaoli@qq.com, ${ }^{*}$ corresponding author
}

Keywords: Fusen herbal tea, spleen deficiency, gastrointestinal tissue

\begin{abstract}
Abstract: Objective: To observe the change ofFusen herbal tea of spleen deficiency rats induced by rhubarb and stomach tissue morphology. Methods: rats were given continuous 17D two times daily intragastric administration of $15 \%$ rhubarb powder suspension ( $5 \mathrm{~mL}$ ) model, divided into large dose small dose of Fusen herbal tea, Fu Tea (half dose source); blank group was given normal drinking water, drinking water of Baohewan and Baohewan group solution. A sufficient amount of solution for the rats drinking water bottle, even changed every day in the 17D solution, the rats were sacrificed at the gastric pathological observation. Results: the rat model of spleen deficiency model, stomach model group pathological changes of histology, compared with the model group, the large and small dose of Fusen herbal tea had no significant damage to the stomach. Conclusion:Fusen herbal tea did great damage to the rhubarb in spleen deficiency rats induced by the stomach.
\end{abstract}

\section{Introduction}

Fusen herbal tea by immortality, chrysanthemum, honeysuckle, licorice, etc. components. The stomach is a major cause of eating habits bad eating restraint, often eat cold drinks or cold food cause. Together with the life rhythm fast, mental pressure big, more easily lead to stomach. So drinking Fusen herbal tea will not cause stomach disease. Therefore, to observe the long-term effects of spleen deficiency model of a large number of drinking Fussen source herbal tea induced by Rhubarb in rats.

\section{Experimental materials}

Fusen herbal tea: by Chinese medicine herb, chrysanthemum, honeysuckle, licorice and other components; rhubarb: purchase of Zhang Zhongjing Pharmaceutical Co., Ltd.; saline: Henan Kelun Pharmaceutical Co., Ltd. is the production, batch C1105052005-2; formaldehyde solution: Yantai both Chemical Co., Ltd. is the production, batch number 20150701 Baohe Pill: Henan Province Wan West Pharmaceutical Co., Ltd. production and batch 141002. 100ml bottle: Zhengzhou glass instrument factory production capacity: 250ml bottles; Zhengzhou glass instrument factory; Electronic scales: Ohaus (Shanghai) Company; 100ml: a Zhengzhou glass instrument factory production.

Rat, SPF, male and female, weighing $160 \sim 180 \mathrm{~g}$, the experimental animal center of Shandong province quality certificate: No 3700540000032.

$10 \%$ formalin fixed solution: $40 \%$ formaldehyde solution with $10 \mathrm{ml}$, distilled water $100 \mathrm{ml}$.

Data analysis using SPSS 17 windows for statistical software, the measurement data between the two groups were compared using one factor analysis of variance, grade data using Ridit analysis. 


\section{Experimental methods}

60 male and female each half rats, rats weighing $160 \sim 180 \mathrm{~g}$, were randomly divided into 5 groups, one group was the control group, 4 rats in rat model of spleen deficiency of rhubarb, spleen deficiency rats each intragastric administration of $15 \%$ rhubarb powder suspension $(5 \mathrm{~mL})$, 17D rats for two times a day, divided high dose small dose of Fusen herbal tea herbal tea, herbal tea (half dose source); blank group were given normal drinking water, drinking water of Baohewan and Baohewan group solution. A sufficient amount of solution for the rats drinking, even daily drinking bottle replacement solution, the rats in the cage were observed daily water intake, feed quantity; weigh.17d fasting (12 h) but the water, 17D rats were killed by cervical dislocation, the stomach and duodenum, the stomach is cut off, the contents make full use of saline wash, and then fixed 24h in stomach and duodenum tissue is placed in 10\% neutral buffered formaldehyde solution, paraffin embedding, slicing, mirror check.

\section{Experimental results}

Weight change of rat model. From the table can be seen, the changes of body weight of rats after drinking water, in 1D group and model group no significant difference $(P>0.05)$, indicating a homogeneous subgroup. On the 16th day, compared with the blank control group compared to model group appeared significantly decreased $(P<0.01)$, compared with the model, the big yellow big and small Ji Liangfu Senyuan herbal tea group can make the body weight of the rats increased significantly $(P<0.05)$, suggesting that Fussen source herbal tea on weight effect is obviously less than that of the model group; and suggests that a large number of short-term drinking Fusen source herbal tea on body weight of rats has little effect. But in the long run, drinking a lot of Fusen source herbal tea can a slight effect on the body weight of rats.

Table 1 Weight change of rat model $(\bar{x} \pm s)$

\begin{tabular}{|c|c|c|c|}
\hline Group & $\mathrm{n}$ & $1^{\mathrm{st}}$ & $16^{\text {th }}$ \\
\hline Blank control group & 10 & $183.70 \pm 18.05$ & $253.01 \pm 18.18^{* *}$ \\
\hline Model group & 10 & $188.63 \pm 16.05$ & $193.20 \pm 18.94$ \\
\hline Baohewan group & 10 & $185.87 \pm 14.97$ & $227.54 \pm 21.97 * *$ \\
\hline high dose of Fusen herbal tea group & 10 & $182.37 \pm 16.91$ & $212.31 \pm 20.03^{*}$ \\
\hline Small dose of Fusen herbal tea group & 10 & $182.73 \pm 10.52$ & $215.06 \pm 24.00^{*}$ \\
\hline high dose of Wang Laoji group & 10 & $185.35 \pm 19.03$ & $200.12 \pm 19.08$ \\
\hline Small dose of Wang Laoji group & 10 & $187.24 \pm 8.16$ & $204.9 \pm 17.96$ \\
\hline
\end{tabular}

Note: compared with model group, ${ }^{*} P<0.05$, ${ }^{* *} P<0.01$

Amount of water and feed. From the table we can see that changes in water intake and feed amount of drinking Fusen herbal tea after rats, and the average value of 17 days can be found in Baohewan group rats than the other group of drinking water source's herbal tea and feed demand more, large yellow, small dose of Fu Tea Senyuan rats the water demand quantity difference; Fusen herbal tea rats than Wanglaoji herbal tea for drinking water and feed demand is slightly less than the two large group Fusen source tea; yellow on the whole, consumption of water and feed the small dose of Fu Senyuan rats herbal tea slightly more than the model group. It is suggested that Fu cool tea on the drinking water source. Effect of feed amount is significantly less than the model group; at the same time that short-term drinking a lot ofFusen herbal tea, has little effect on the rat diet and water intake, but a large number of long-term drinking herbal tea can be a source's effect on rat food intake and water intake. 
Table 2 Amount of water and feed $(\bar{X} \pm s)$

\begin{tabular}{|c|c|c|c|c|c|c|c|}
\hline Way & $\begin{array}{l}\text { Blank control } \\
\text { group }\end{array}$ & Model group & $\begin{array}{c}\text { Baohewan } \\
\text { group }\end{array}$ & $\begin{array}{l}\text { high dose of } \\
\text { Fusen } \\
\text { herbal tea in } \\
\text { group }\end{array}$ & $\begin{array}{l}\text { Small dose of } \\
\text { Fusen herbal } \\
\text { tea in group }\end{array}$ & $\begin{array}{l}\text { high dose of } \\
\text { Fusen herbal } \\
\text { tea in group }\end{array}$ & $\begin{array}{l}\text { Small dose of } \\
\text { Fusen herbal tea } \\
\text { in group }\end{array}$ \\
\hline $\begin{array}{l}\text { water average } \\
(\mathrm{ml})\end{array}$ & $339.3 \pm 27.0$ & $244.3 \pm 22.0$ & $280.0 \pm 20.5$ & $265.2 \pm 19.5$ & $268.1 \pm 19.5$ & $252.3 \pm 19.8$ & $260.0 \pm 19.6$ \\
\hline $\begin{array}{l}\text { Feed average } \\
\text { (g) }\end{array}$ & $193.8 \pm 11.5$ & $122.4 \pm 9.3$ & $151.5 \pm 9.2$ & $139.9 \pm 6.9$ & $140.7 \pm 8.8$ & $135.3 \pm 6.0$ & $132.4 \pm 5.4$ \\
\hline
\end{tabular}

Note: There were ten rats in each group

\section{Influence of the organization form of the Department}

The stomach tissue of rats, formalin fixed, sliced, stained and observed pathological changes of rat stomach. The results are shown in Table 3

Table 3 Effects of rhubarb on the morphology of gastric tissue in rats with spleen deficiency

\begin{tabular}{|c|c|c|c|c|c|}
\hline Group & $\mathrm{n}$ & - & + & ++ & +++ \\
\hline Blank control group & 10 & 10 & 0 & 0 & 0 \\
\hline Model group & 10 & 0 & 1 & 3 & 6 \\
\hline Baohewan group & 10 & 3 & 2 & 2 & 3 \\
\hline high dose of Fusen herbal tea group & 10 & 4 & 1 & 2 & 3 \\
\hline Small dose of Fusen herbal tea group & 10 & 1 & 2 & 3 & 4 \\
\hline high dose of Wang Laoji group & 10 & 2 & 2 & 2 & 4 \\
\hline Small dose of Wang Laoji group & 10 & 10 & 0 & 0 & 0 \\
\hline
\end{tabular}
gastric mucosa epithelial cells occasionally see epithelial cells atrophy and necrosis falls off phenomenon; "+ +" shallow layer of gastric mucosa epithelial cell atrophy, degeneration, some cells appeared necrosis; "+ + +" diffuse gastric mucosa epithelial cells atrophy, necrosis and exfoliation.

From the table can be seen, the blank control group, gastric mucosa had no obvious abnormalities; a large number of long-term drinking Fussen source herbal tea will of gastric mucosa in spleen deficiency rats have a slight impact, gastric mucosa occasionally part of the region appeared in principal cells, reduce the number of cell wall, reduce the number of gastric body; Baohewan rats has been gradually restored to normal rat;, small dose Senyuan Fu Tea rats gastric mucosal changes relatively small. By Ridit test, and blank control group, each group of data without significant difference thatFusen herbal tea on gastrointestinal function in rats with deficiency of the spleen without significantly influenced.

\section{Observation of gastric tissue in rats under light microscope}

Source section of the shallow layer of thickness of the muscular layer of the structural integrity of the gastric mucosal histopathological observation: blank control group rats gastric mucosa, mucosal epithelial cells arranged in neat rows, glandular epithelial cells is basic and normal, natural layer with no congestion and inflammatory cell infiltration, mucosal integrity; Baohe Pill group rat gastric mucosa and gastric mucosa epithelial cells recovered to normal rats; rhubarb induced gastric spleen deficiency model group rats gastric mucosa epithelial cells atrophy, degeneration, some cells appeared necrosis fall off; large yellow Fussen source herbal tea high dose group of gastric mucosa 
epithelial cells atrophy, necrosis, shedding phenomenon less; large yellow Fussen herbal tea small dose group occasionally epithelial cells atrophy and necrosis falls off phenomenon.

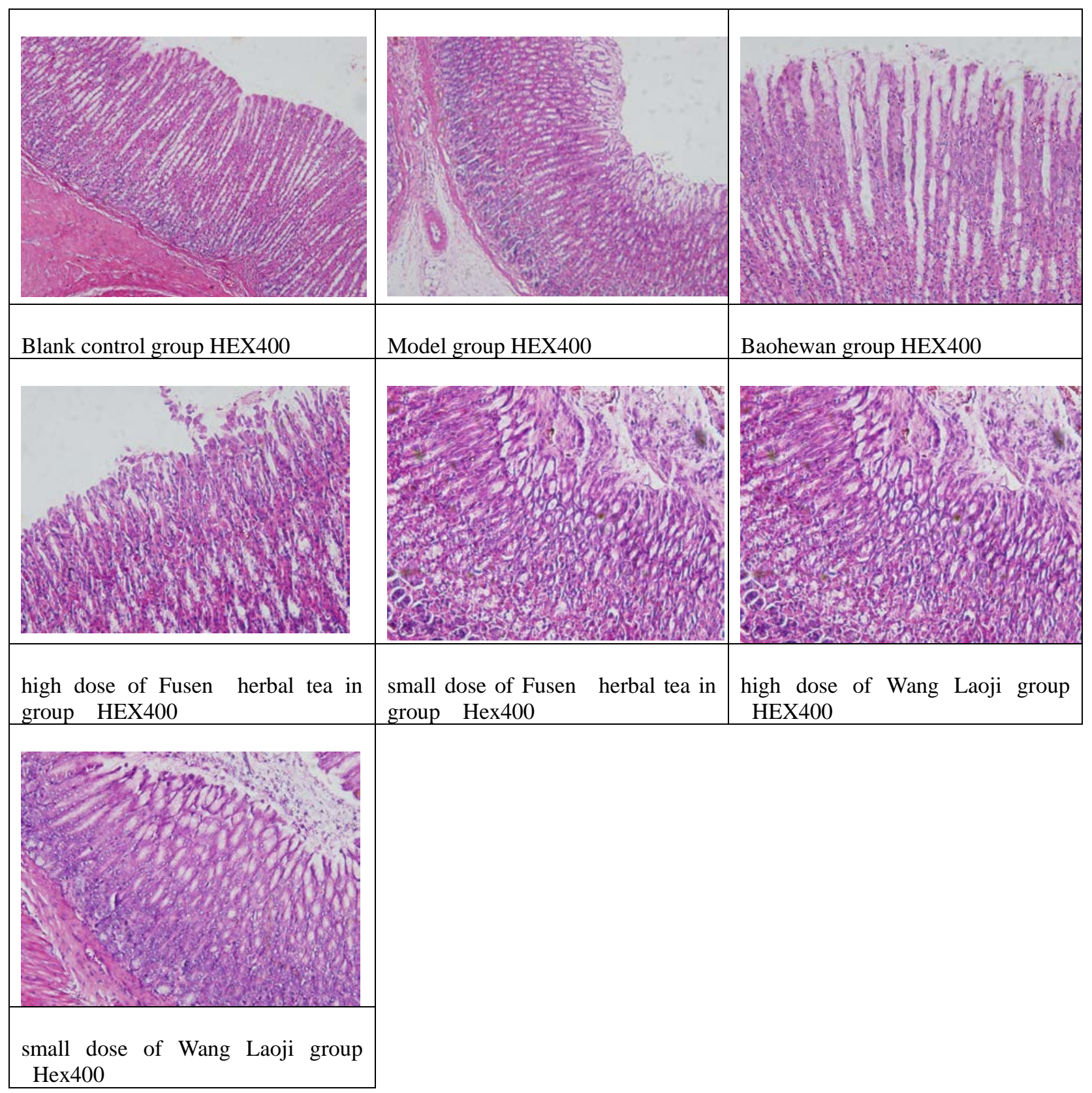

\section{Conclusions}

Modern city for dinner irregular diet lead to stomach disease proportion is rising year by year, the weakness of the spleen and stomach population is becoming more and more popular, and $\mathrm{Fu}$ Senyuan herbal tea containing many cool traditional Chinese medicine that will aggravate the degree of spleen deficiency group, but after the experiment show that prompt Fussen source herbal tea on weight were less affected in the Wong Lo Kat; and suggests that a large number of short-term drinking Fusen herbal tea, on body weight of rats has little effect, but long-term drinking a lot of Fussen source herbal tea can be a slight effect on the body weight of rats, which amount of water intake and feed rats and consistent trend. ThatFusen herbal tea of spleen deficiency rats without obvious adverse effects.

From the experimental microstructure showed that the blank control group rat gastric mucosa had no obvious abnormalities; Baohewan group rats have been gradually returned to normal level in rats; however, a small dose of Fu Group rat gastric mucosa Liangcha source change is relatively 
small; although the long-term drinking herbal tea will have a slight impact source forsen on gastric mucosa of spleen deficiency rats, but no gastric mucosa of the rats in the model group the change is relatively large. At the same time, through the test of Ridit, compared with the blank control group, there was no significant difference between each group of data source and Fu tea; histopathology showed that the blank control group of rat gastric mucosa structural integrity of epithelial cells arranged orderly, epithelial cells of normal glands no, the lamina propria congestion and inflammatory cell infiltration, muscularis mucosa integrity; Baohewan rats gastric mucosal thickness and gastric mucosal epithelial cells restored to the normal level in rats induced by Rhubarb; Stomach spleen deficiency model rats shallow gastric mucosa epithelial cells atrophy, degeneration, with some cells appeared necrosis; Fussen source herbal tea large and small dose group I see epithelial cells atrophy and necrosis falls off phenomenon. Fusen herbal tea on stomach function of spleen deficiency rats without significantly influenced.

Ran due to source Fussen herbal tea containing cold medicine, but according to the weight, food intake and water intake did not find related increase model of spleen deficiency, histologically from further proved Fusen herbal tea on the stomach without obvious adverse effects. Fusen herbal tea on gastrointestinal function in rats with deficiency of the spleen without obvious adverse effects. The experiments show, Fussen source herbal tea on gastrointestinal function in rats with deficiency of the spleen without obvious adverse effect. This experiment provides the application of Fusen herbal tea the experimental basis.

\section{References}

[1]L. Chen, Wang Chuijie. Comparison of gastric mucosal damage in rats with spleen deficiency syndrome (3) [J].:127-129. (3).2013,21

[2]Yan Shengguo, Yu Hongwu, Yong Lihua, Yang Hongsheng, Wei Yanming, Weichangning on reserpine induced rat model of spleen deficiency [J]. China animal experiments Journal of. 2011, 9 (3): 242-245.

[3]Fang Xiongwu, Hongyan, Hainan and rhubarb on plasma of rats with spleen qi deficiency growth chalone and gastric mucosal injury [J]. Chinese medicine forum.2010,25 (1): 35-37.

[4]Huang Qiuyun, Xu Jun. Eight pork stomach spleen deficiency of rats diet effects on gastric ulcer [J]. Zhejiang Journal of traditional Chinese Medicine.2013,48 (11):836-839.

[5]Manuela in Pan Zhiqiang, coincident Hou Li. Different methods induced experimental gastric ulcer in mice of different syndromes in the same disease [J]. Journal of Hunan Chinese medicine. 2008,24 (1): 70-78.

[6]Miao Mingsan, Zhou Hongyan, Li Ping ruffian. Full Xiao granule effect on gastrointestinal peristalsis of mice [J],.2000.2,13 traditional Chinese Medicine Research (1): 34-35.

[7]Ma Fengyu, Xiao Yijia, Huang Rongrong, Tyvek. Shu Wei Ling on rat gastrointestinal function and protective effect on gastric mucosa [J], massage and rehabilitation medicine, 2011.6 (53):3-5.

[8]Hershey P, Xi Ping, Liao Xuezhen, Chen Muyuan. Experimental study on the fhirta Decoction of rhubarb spleen deficiency gastrointestinal motor function in mice [J]. Pharmacy today, 2012: 22 (7):398-401.

[9]Shen Libo, Qian Huinan. Experimental research method of model of spleen deficiency China overview [J]. Journal of Medicine 2005:12 (1):93-95. 